\title{
Dietary intakes of flavan-3-ols and cardiovascular health: a field synopsis using evidence mapping of randomized trials and prospective cohort studies
}

Gowri Raman $^{1^{*}}$ D, Marissa Shams-White ${ }^{1,2}$, Esther E. Avendano ${ }^{1}$, Fan Chen ${ }^{1,2}$, Janet A. Novotny ${ }^{3}$ and Aedin Cassidy ${ }^{4}$

\begin{abstract}
Background: There is considerable interest in the impact of increased flavan-3-ol intake on cardiovascular disease (CVD) and diabetes outcomes. Through evidence mapping, we determined the extent of the evidence base to initiate a future systematic review investigating the impact of flavan-3-ol intake on CVD and diabetes outcomes.

Methods: We developed a research protocol, convened a technical expert panel (TEP) to refine the specific research questions, conducted a systematic search in multiple databases, double-screened abstracts and full-text articles, performed data extractions, and synthesized the data. We focused on randomized controlled trials (RCTs) and prospective cohort studies which assessed intakes of flavan-3-ol from foods, beverages, and supplement/extract sources on biomarkers and clinical outcomes of CVD and diabetes.

Results: Of 257 eligible articles, 223 and 34 publications contributed to 226 RCTs and 39 prospective cohort studies, respectively. In RCTs, the most frequently studied interventions were cocoa-based products (23.2\%); berries (16.1\%); tea in the form of green tea (13.9\%), black tea (7.2\%), or unspecified tea (3.6\%); and red wine (11.2\%). Mean total flavan-3-ol intake was highest in the cocoa-based trials $(618.7 \mathrm{mg} /$ day) and lowest in the interventions feeding red wine (123. $7 \mathrm{mg} /$ day). The most frequently reported outcomes were intermediate biomarkers including serum lipid levels (63.4\%), blood glucose (50.9\%), blood pressure (50.8\%), flow-mediated dilation (21.9\%), and high-sensitivity C-reactive protein (21.9\%). The included 34 prospective cohort studies predominantly examined exposures to flavan-3-ols (26\%), cocoa-based products (23.2\%), berries (16.1\%), and green tea (13.9\%) and CVD incidence and mortality.

Conclusion: Through a systematic, evidence-based approach, evidence mapping on flavan-3-ol intake and CVD outcomes demonstrated sufficient data relating to flavan-3ol intake and biomarkers and clinical outcomes of CVD and diabetes. The current evidence base highlights the distribution of available data which both support the development of a future systematic review and identified the research need for future long-term RCTs.
\end{abstract}

Systematic review registration: At present, evidence mapping is not eligible for registration on the international prospective register of systematic reviews (i.e., PROSPERO).

Keywords: Cardiovascular disease, Diabetes, Lipids, Flavan-3-ols, Evidence mapping, Cocoa, Tea, Berries, Cinnamon, Red wine

\footnotetext{
* Correspondence: graman@tuftsmedicalcenter.org

${ }^{1}$ Tufts Center for Clinical Evidence Synthesis, Institute for Clinical Research and Health Policy Studies, Tufts Medical Center, Box 63, 800 Washington Street, Boston, MA 02111, USA

Full list of author information is available at the end of the article
}

(c) The Author(s). 2018 Open Access This article is distributed under the terms of the Creative Commons Attribution 4.0 International License (http://creativecommons.org/licenses/by/4.0/), which permits unrestricted use, distribution, and reproduction in any medium, provided you give appropriate credit to the original author(s) and the source, provide a link to the Creative Commons license, and indicate if changes were made. The Creative Commons Public Domain Dedication waiver (http://creativecommons.org/publicdomain/zero/1.0/) applies to the data made available in this article, unless otherwise stated. 


\section{Background}

Dietary flavonoids represent a diverse range of polyphenolic compounds that occur naturally in plant foods. Their structural complexity has led to their sub-classification as flavonols, flavones, flavanones, flavan-3-ols (and their oligomers, pro(antho)cyanidins), isoflavones, and anthocyanins [1]. Although interest in the relationship between these bioactive constituents and cardiovascular disease (CVD) and diabetes risk is growing, their specific contribution to any health effects and the underlying mechanisms by which they act is an area of active research $[2,3]$.

To date, the sub-class that has received the most attention is flavan-3-ols, although most of the studies to date focus on two of the dietary sources, tea and chocolate. A number of meta-analyses have been published on the existing population-based evidence for these individual foods [1, 4-8]. Additionally, it is important to note that any health benefit from intake of tea or chocolate may be attributed to other bioactive constituents beyond flavan-3-ols and flavonoids, including other phenolics, caffeine, theobromine [9-11]. To date, in randomized controlled trials (RCTs) and prospective studies, although available findings on flavan-3-ols-rich foods are promising, dose-responses varied and the overall results are mixed. Potential adverse effects of flavan-3-ol extracts and supplements are also reported in literature $[12,13]$. Studying a broad range of food sources rich in flavan-3-ols will help to further elucidate the potential health effects of flavan-3-ols per se to improve cardiometabolic health.

Evidence mapping is a tool used to systematically identify, organize, and summarize the quantity and focus of scientific evidence on a broad range of topics. Through evidence mapping, we determine the extent of the evidence base examining the impact of flavan-3-ol intake on CVD and diabetes outcomes and associated risk factors in RCTs and prospective cohort studies. The overall goal was to provide recommendations for future research and to identify if there is sufficient evidence to date to support initiating a full systematic review.

\section{Methods}

\section{Description of evidence mapping}

Evidence mapping is a tool to identify well-researched areas as well as gaps in evidence. It is often conducted to evaluate evidence on a broad topic before embarking on a full systematic review [14, 15]. Evidence mapping describes the characteristics of eligible literature typically in tabular forms and figures. Creating an evidence map usually involves the following steps: identifying a broad research area, defining key questions and variables and formulating a framework, engaging key stakeholders, conducting a systematic literature search, selecting studies for inclusion according to a priori eligibility criteria, and reporting descriptive results in tabular formats. In contrast to conducting a systematic review, evidence mapping does not necessitate detailed extraction, the assessment of risk of bias, and quantitative synthesis of results from eligible studies.

\section{Scope of the evidence map}

Currently, there is no prescribed methodological or reporting standard (i.e., for evidence mapping) and, therefore, we followed a series of logical steps in creating this evidence map. First, we assembled a review team and developed a protocol; identified and convened a technical expert panel (TEP) with content expertise, methodological expertise, or both; refined specific research questions with the input of the TEP; and conducted a systematic search in multiple databases. We then double-screened abstracts and full-text articles, performed data extractions, and synthesized data in tabular forms. The data was used to identify areas within the topic of flavan-3-ols and cardiovascular outcomes with a paucity of research that are ideal focuses for future studies, as well as well-researched topics where there is sufficient evidence to warrant a full systematic review.

\section{Review team and development of a protocol}

Our review team was comprised of two nutrition researchers with expertise in flavan-3-ols, one methodologist, and three research assistants with an interest and training in nutrition research. First, we identified preliminary key questions and study eligibility criteria through discussions of Populations, Interventions/Exposures, Comparators, Outcomes, and Study designs or Settings (PI(E)COS) of interest and developed the analytic framework (Fig. 1). Next, we utilized the United States Department of Agriculture (USDA) monomer and procyanidin databases or Phenol-Explorer databases to identify commonly consumed foods and beverages that have the highest flavan-3-ol content-either as monomers or polymers-and assembled a comprehensive list of them in an Excel $^{\text {Ts }}$ spreadsheet [16-18]. We did not search for foods that have low flavan-3-ol content but are consumed at a high frequency. A detailed search strategy and protocol was then developed outlining key questions and the scope of the review. This review was submitted to the International Life Sciences Institute (ILSI) bioactive committee for their input, but we retained the independence to decide whether or not to incorporate any suggestions received from the committee.

\section{Technical expert panel}

Our review team identified and convened a multidisciplinary TEP, which included four flavan-3-ol researchers from the USDA, academic centers in North America and the UK; two methodologists with expertise in nutrition research; and a cardiologist. The TEP provided input to help refine the 


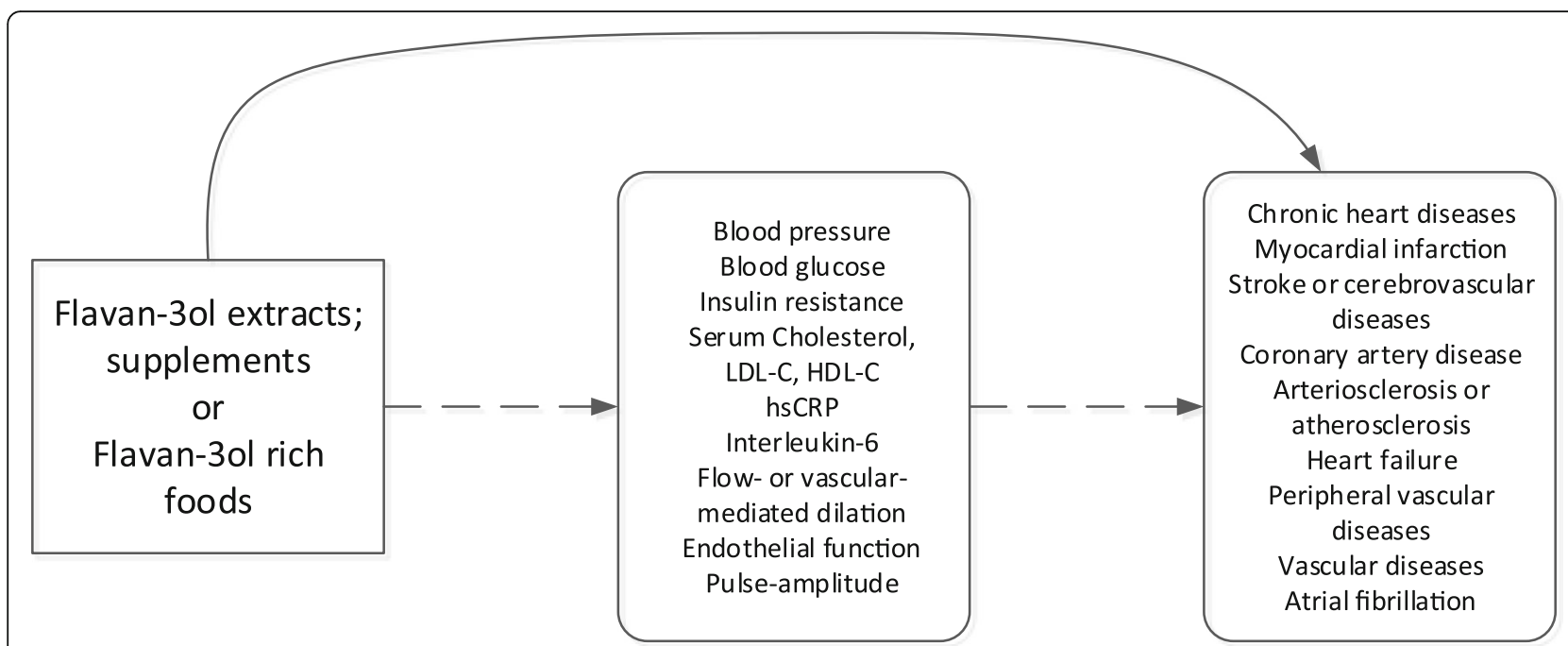

Fig. 1 Analytic framework for evaluating the association between flavan-3-ol and outcomes. Legend: Analytic framework displays the potential association pathway between flavan-3-ol and outcomes of interest

questions and protocol, identify important issues, and define parameters for the mapping of evidence. The review team also asked the TEP to suggest additional search strategy terms. See Additional file 1: Table S1 for a list of TEP members and affiliations.

\section{Search strategy}

A comprehensive search of the scientific literature was conducted in MEDLINE ${ }^{\oplus}$, the Cochrane Central Trials Registry ${ }^{\oplus}$, and Commonwealth Agricultural Bureau (CAB) from inception through April 26, 2016. The reference lists of any previously published systematic reviews on flavonoids and health identified by the review team or TEP were hand-searched to identify any additional studies not captured by the database searches.

To capture interventions of interest, we combined terms of monomer and proanthocyanidins for flavan-3-ols, foods, beverages, and spices (e.g., cinnamon) rich in flavan-3-ols with the outcomes of interest including any cardiovascular outcomes and intermediate biomarkers, inflammatory markers, and endothelial function. The search strategy included terms for the study designs of interest (i.e., randomized trials and prospective cohort studies) and was limited to those conducted in humans (see Additional file 1: Table S2 for the complete search strategy). No language restriction was applied during the search process.

\section{Study selection}

Our review team used an iterative process to establish study eligibility criteria based on the aforementioned PI(E)COS, detailed in Tables 1 and 2. Using these criteria, we assessed abstracts and/or titles of citations identified from literature searches for inclusion, retrieved full-text articles of potentially relevant citations, and re-assessed the articles for inclusion. Articles published in the English language were selected. Both abstract and full-text screenings were conducted in duplicate, with conflicts resolved by reconciliation with the whole research team in weekly group meetings. The project lead reconfirmed all rejected full-text articles. Additionally, content experts were consulted through emails for questions that arose in weekly group meetings.

\section{Data extraction}

In discussion with nutrition experts within the review team, data was extracted into customized forms in the Systematic Review Data Repository (SRDR) online system (https://srdr.ahrq.gov). The forms were designed to capture all elements relevant to the descriptions on study population characteristics, enrolled and analyzed sample sizes, study design features, interventions and comparators, and relevant outcomes. If flavan-3-ol levels were not explicitly reported in the paper, where possible, data extractors estimated flavan-3-ol intake levels using either the USDA monomer and proanthocyanidins or Phenol-Explorer databases [16-18]. We also extracted qualitative outcomes (i.e., direction of association and statistical significance). The forms were piloted on several studies, revised, and optimized as necessary by the project lead. One team member extracted each study's data. A second team member confirmed interventions and outcome data entries at the time of data analysis.

\section{Data synthesis}

All included studies were summarized in narrative form and in summary tables that tabulated the important 
Table 1 Inclusion and exclusion criteria

\begin{tabular}{|c|c|}
\hline Inclusion criteria & Exclusion criteria \\
\hline Populations: humans only & Populations: those including: \\
\hline Adults $18+$ years of age & Non-human subjects (e.g., in vitro, cell, stem cell, or animal studies) \\
\hline \multicolumn{2}{|l|}{ Healthy adults } \\
\hline Adults at an increased risk for cardiovascular disease (CVD) & Children and adolescents ( $<18$ years old) \\
\hline Adults who had existing CVD & Pregnant or lactating women \\
\hline Cancer survivors who were cancer-free at the time of the study & Adults with prior organ transplantation \\
\hline \multirow[t]{4}{*}{ All elderly unless clear disease (e.g., renal disease) } & $\begin{array}{l}\text { Adults with chronic, inflammatory, or autoimmune disease conditions } \\
\text { (e.g., rheumatoid arthritis, polycystic ovarian syndrome, } \\
\text { chronic kidney disease) }\end{array}$ \\
\hline & $100 \%$ smokers \\
\hline & Participants in concurrent cancer or exercise trials \\
\hline & Studies with $<10$ subjects \\
\hline Interventions: & Interventions: \\
\hline $\begin{array}{l}\text { Foods, beverages, supplements, or extracts that described or defined } \\
\text { flavan-3-ol content either as monomers or polymers }\end{array}$ & $\begin{array}{l}\text { Did not evaluate a specific intervention } \\
\text { (e.g., spice blend, dietary patterns rich in flavan-3-ol food sources) }\end{array}$ \\
\hline \multirow[t]{2}{*}{$\begin{array}{l}\text { Example foods: fruits (e.g., apples, berries, grapes), dark chocolate, } \\
\text { cocoa, teas, red wine) }\end{array}$} & $\begin{array}{l}\text { Foods with no or low flavan-3-ol content } \\
\text { (e.g., white chocolate, raisins) }\end{array}$ \\
\hline & $\begin{array}{l}\text { Foods with insufficient data to allow a reasonable estimation } \\
\text { of flavan-3-ol content using standardized databases [16-18] }\end{array}$ \\
\hline $\begin{array}{l}\text { Studies must quantify flavan-3-ols consumed or provide sufficient data } \\
\text { that allowed estimation of the flavan-3-ol content via standardized } \\
\text { databases }^{1}[16-18]\end{array}$ & $\begin{array}{l}\text { Did not specify the type of wine (e.g., white, red, rice), alcohol, } \\
\text { or chocolate (e.g., dark, white, or milk chocolate) in their } \\
\text { questionnaires and/or reported results }\end{array}$ \\
\hline Comparators of interest: & Comparators of interest: \\
\hline Low or no flavan-3-ol content, including placebos & $\begin{array}{l}\text { Studies that did not differentiate flavan-3-ol content } \\
\text { (e.g., a study evaluating the effects of alcohol comparing } \\
\text { red wine vs. de-alcoholized red wine) }\end{array}$ \\
\hline Outcomes of interest: & Outcomes of interest: \\
\hline Key clinical and intermediate outcomes (Table 2$)^{2}$ & $\begin{array}{l}\text { Clinical and intermediate outcomes of interest (Table 2) that do } \\
\text { not meet the minimum follow-up requirements }\end{array}$ \\
\hline Study designs of interest: & Study designs of interest: \\
\hline Randomized controlled trials (RCTs) & Cross-sectional studies \\
\hline \multirow[t]{5}{*}{ Prospective cohort studies } & Case reports \\
\hline & Case series \\
\hline & Retrospective studies \\
\hline & Systematic reviews and meta-analyses ${ }^{3}$ \\
\hline & $\begin{array}{l}\text { Reviews, lectures, opinion articles, news articles, proposed studies } \\
\text { (i.e., no results reported) }\end{array}$ \\
\hline
\end{tabular}

${ }^{1}$ For all interventions of interest when flavan-3-ols were not fully reported in an article, we utilized the recent USDA monomer and procyanidin databases or Phenol-Explorer databases to estimate flavan-3-ol content-either as monomers or procyanidin based on the intake values reported in each article.

${ }^{2}$ When outcomes were reported at multiple time points, only baseline and final follow-up results were extracted

${ }^{3}$ Though systematic reviews and meta-analyses were excluded during the screening process, their references were screened for any additional, relevant studies

features of the study populations, designs, interventions, outcomes, and results. Studies were also summarized qualitatively in tabular form and graphically using heat maps.

\section{Results}

We identified 3194 abstracts examining flavan-3-ol intakes and cardiovascular outcomes (including MI, stroke) and a range of CVD risk factors. Of these, 536 (16.8\%) abstracts met the broad eligibility criteria and were retrieved and re-examined as full-text articles; 257 of the full-text articles (223 RCTs and 34 prospective studies) were eligible for data entry into the final database (Fig. 2). Of the 257 articles, 223 publications contributed to 226 RCTs and 34 publications contributed to 39 prospective observational cohort studies (i.e., some articles reported multiple studies), and eligible studies are listed in the Additional file 1. 
Table 2 Included outcomes of interest and minimum follow-up cutoffs

o Cardiovascular disease (CVD) clinical outcomes ( $\geq 6$ months follow-up), including acute coronary syndrome, angina, arteriosclerosis, atherosclerosis, atrial fibrillation, cerebrovascular disorders, coronary artery disease, heart failure, myocardial infarction, peripheral vascular disease, and venous thromboembolism ${ }^{1}$

- Serum lipids ( $\geq 3$ weeks follow-up),including blood pressure (BP), diastolic BP (DBP), low-density lipoprotein (LDL), high-density lipoprotein (HDL), systolic BP (SBP), total cholesterol, triglycerides

- Inflammatory markers ( $\geq 3$ weeks follow-up), including high-sensitivity C-reactive protein (hs-CRP), intercellular adhesion molecule 1 (ICAM-1), interleukins, vascular cell adhesion molecule 1 (VCAM-1)

- Metabolic parameters ( $\geq 1$ week follow-up), including blood glucose, diabetes, hemoglobin $\mathrm{A} 1 \mathrm{C}(\mathrm{HbA} 1 \mathrm{C})$, homeostatic model assessment and insulin resistance (HOMA-IR), insulin resistance, quantitative insulin sensitivity check index (QUICKI)

- Evaluation of endothelial function (any study length, including acute studies), using Endo-PAT, flow-mediated dilation (FMD), pulse-amplitude, pulse wave analysis, pulse wave velocity

${ }^{1}$ When outcomes were reported at multiple time points, the results reported at baseline and the final follow-up were extracted

\section{Description of RCTs}

The 223 articles were published between March 1985 and April 2016 and contributed to 226 RCTs in the database (i.e., three publications contained two RCTs each) (Fig. 3). The top three regions where the RCTs were conducted were Western Europe (38.6\%), North America (26.5\%), and Asia (13.0\%). The majority of RCTs were parallel study designs (62.3\%), and the remaining were $(37.7 \%)$ crossover studies. Regarding blinding, 55.6\% of the RCTs were double-blinded, $17.9 \%$ were single-blinded, $11.2 \%$ were not blinded, and $15.3 \%$ did not explicitly report on blinding. Over half of the RCTs were conducted among adults $\geq 50$ years of age (56.1\%); $57.0 \%$ included predominantly metabolically at-risk adults; $29.2 \%$ included healthy adults (i.e., adults without any risk factors for CVD or without existing CVD); $4.9 \%$ included adults with existing CVD; $4.9 \%$ included adults with more than one of the

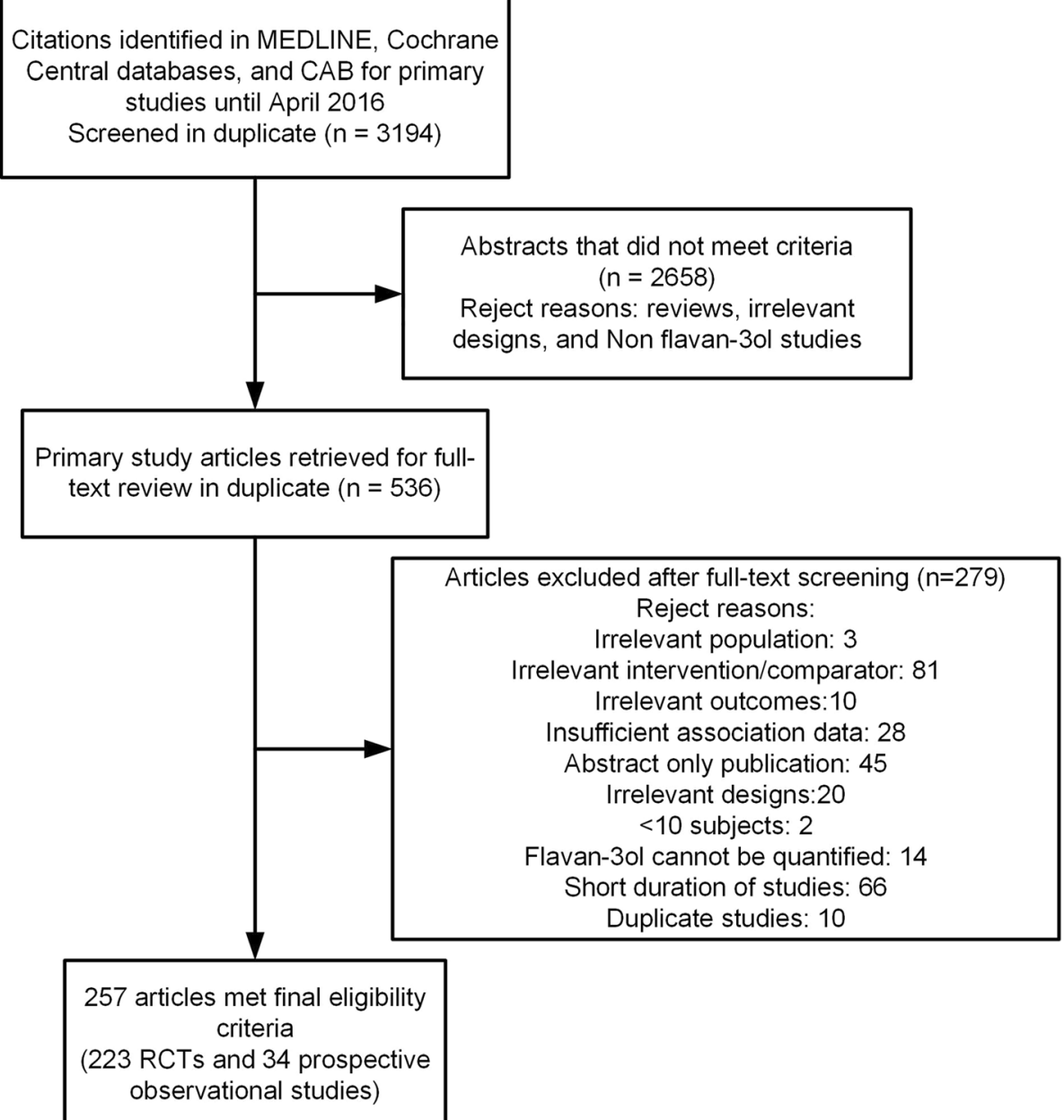

Fig. 2 Study Flow Diagram. Legend: Abstracts identified $(n=3194)$; Abstracts not meeting criteria $(n=2658) ;$ Full-text articles excluded after screening $(n=279)$; Full-text articles meeting study eligibility criteria $(n=257)$; Eligible randomized controlled trials $(n=223)$ and prospective observational studies $(n=34)$. RCTs $=$ randomized controlled trials 


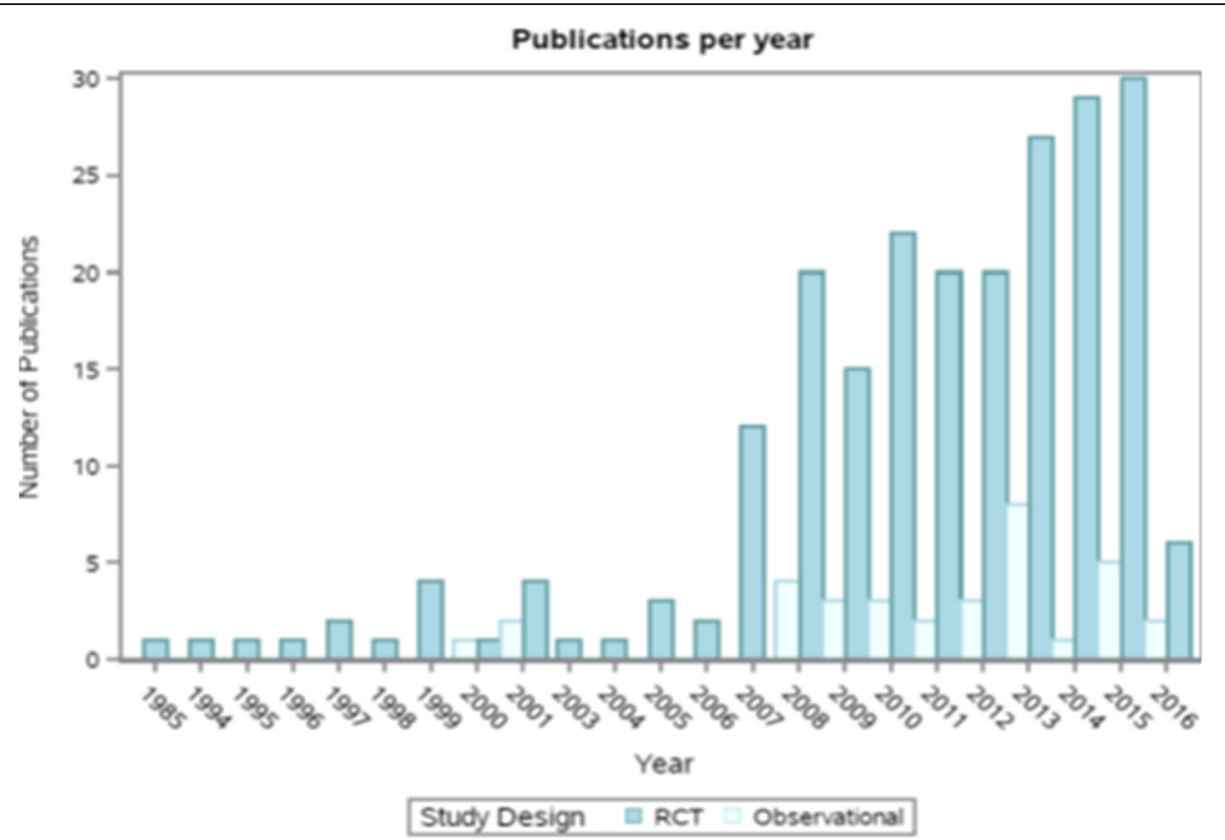

Fig. 3 Bar graph displaying the mean number of RCT and observational study flavan-3-ols articles published per year between 1985 and 2016. $\mathrm{RCT}=$ randomized controlled trial

aforementioned categories; and 4.0\% included adults with other conditions at baseline.

\section{Included interventions of interest}

The most frequently studied intervention in RCTs was cocoa-based products $(23.2 \%)$, consumed as dark chocolate, cocoa, or added cocoa polyphenols. The second most frequently studied intervention was tea in the form of green tea (13.9\%), black tea (7.2\%), or unspecified tea (3.6\%), followed by berries and red wine in 16.1 and $11.2 \%$ of the RCTs, respectively. Less than $10 \%$ of the RCTs included other interventions such as apples, cinnamon, cranberries, flavan-3-ol monomer or proanthocyanidin supplementation, grapes, grape seed, plums, and mixed interventions of interest. Table 3 illustrates the evaluation of these interventions.

Mean total flavan-3-ol intake varied across interventions. It was highest for RCTs administering chocolate $(618.7 \mathrm{mg} /$ day $)$, green tea $(473.4 \mathrm{mg} /$ day $)$ or black tea ( $256.2 \mathrm{mg} /$ day), apples (444.1 mg/day), flavan-3-ol monomers $(350.7 \mathrm{mg} /$ day), berries $(250 \mathrm{mg} /$ day $)$, and red wine (123.7 $\mathrm{mg} /$ day). Across interventions, the average study duration ranged between 4 and 17 weeks. A detailed description of reported or estimated flavan-3-ols is tabulated in the Additional file 1.

\section{Outcomes of interest}

The most frequently reported outcomes in RCTs of berries, cocoa-based products, green tea, and wine were blood glucose $(50.9 \%)$, blood pressure (BP) (50.8\%), serum lipid levels (63.4\%), flow-mediated dilation (FMD) (21.9\%), and high-sensitivity C-reactive protein (hs-CRP) (21.9\%) (Figs. 4 and 5). In addition, cinnamon trials frequently reported blood glucose and blood lipid outcomes, grape seed trials reported FMD and hs-CRP, and cranberry trials reported hs-CRP. The sample sizes per trial ranged from 42 to 70 subjects. Though trials overall were more likely to report improvements in evaluated outcomes after the intervention as compared with the control (Additional file 1), within each group (compared to baseline) statistical significance was reached in $<50 \%$ of the studies and between groups (intervention vs. control) statistical significance was reached in $<25 \%$ of the studies.

Descriptive data on additional outcomes included insulin resistance or homeostatic model assessment and insulin resistance (HOMA-IR) (18.9\%), oxidized low-density lipoprotein (LDL) (12.1\%), pulse-wave velocity (14.3\%), intercellular adhesion molecule 1 (ICAM-1) (11.6\%), vascular cell adhesion molecule 1 (VCAM-1) (12.1\%), interleukin-6 (12.9\%), tumor necrosis factor alpha (TNF-alpha) (11.2\%), and other outcomes $(<10 \%)$.

\section{Description of cohort studies}

The 39 prospective cohort studies (in 34 publications) represented 47 adjusted models. The cohort studies were conducted in Europe (16 studies), the USA (10 studies), Asia (7 studies), and Australia (1 study). The exposures examined included total dietary flavan-3-ol intake (9 
Table 3 Descriptive characteristics of eligible RCTs

\begin{tabular}{|c|c|c|c|}
\hline & Total N (\%) & RCT parallel $N(\%)$ & RCT crossover $N(\%)$ \\
\hline$N$ publications & $223(100.00)$ & $139(62.33)$ & $84(37.67)$ \\
\hline \multicolumn{4}{|l|}{ Blinding } \\
\hline Double-blind & $124(55.61)$ & $87(62.59)$ & $37(44.05)$ \\
\hline Single-blind & $40(17.94)$ & $19(13.67)$ & $21(25.00)$ \\
\hline Not blinded & $25(11.21)$ & $14(10.07)$ & $11(13.10)$ \\
\hline Not reported & $34(15.25)$ & $19(13.67)$ & $15(17.86)$ \\
\hline \multicolumn{4}{|l|}{ Mean age } \\
\hline$<50$ years & $98(43.95)$ & $57(41.01)$ & $41(48.81)$ \\
\hline$\geq 50$ years & $125(56.05)$ & $82(58.99)$ & $43(51.19)$ \\
\hline \multicolumn{4}{|l|}{ Baseline health } \\
\hline Healthy & $65(29.15)$ & $29(20.86)$ & $36(42.86)$ \\
\hline At risk for CVD & $127(56.95)$ & $91(65.47)$ & $36(42.86)$ \\
\hline Existing CVD & $11(4.93)$ & $5(3.60)$ & $6(7.14)$ \\
\hline Mixed & $11(4.93)$ & $6(4.32)$ & $5(5.95)$ \\
\hline Other & $9(4.04)$ & $8(5.76)$ & $1(1.19)$ \\
\hline \multicolumn{4}{|l|}{ Study region } \\
\hline Africa & $2(0.90)$ & $0(0.00)$ & $2(2.38)$ \\
\hline Asia & $29(13.00)$ & $28(20.14)$ & $1(1.19)$ \\
\hline Australia & $12(5.38)$ & $6(4.32)$ & $6(7.14)$ \\
\hline Eastern Europe & $5(2.24)$ & $5(3.60)$ & $0(0.00)$ \\
\hline Western Europe & $86(38.57)$ & $38(27.34)$ & $48(57.14)$ \\
\hline Middle East & $24(10.76)$ & $23(16.55)$ & $1(1.19)$ \\
\hline North America & $59(26.46)$ & $34(24.46)$ & $25(29.76)$ \\
\hline South America & $5(2.24)$ & $4(2.88)$ & $1(1.19)$ \\
\hline Mixed & $1(0.45)$ & $1(0.72)$ & $0(0.00)$ \\
\hline \multicolumn{4}{|l|}{ Intervention } \\
\hline Apple & $4(1.79)$ & $1(0.72)$ & $3(3.57)$ \\
\hline Berries & $36(16.14)$ & $24(17.27)$ & $12(14.29)$ \\
\hline Black tea & $16(7.17)$ & $9(6.47)$ & 7 (8.33) \\
\hline Chocolate & $52(23.21)$ & $27(19.42)$ & 25 (29.76) \\
\hline Cinnamon & 18 (8.07) & $17(12.23)$ & $1(1.19)$ \\
\hline monomers & $9(4.04)$ & $6(4.32)$ & $3(3.57)$ \\
\hline polymers & $1(0.45)$ & $1(0.72)$ & $0(0.00)$ \\
\hline Grape & 7 (3.14) & $4(2.88)$ & $3(3.57)$ \\
\hline Grape seed & $13(5.83)$ & $9(6.47)$ & $4(4.57)$ \\
\hline Green tea & $31(13.90)$ & $26(18.71)$ & $5(5.95)$ \\
\hline Mixed/other & $3(1.35)$ & $3(2.16)$ & $0(0.00)$ \\
\hline Tea & $8(3.59)$ & $4(2.88)$ & $4(4.76)$ \\
\hline Wine & $25(11.21)$ & $8(5.76)$ & 17 (20.24) \\
\hline
\end{tabular}

CVD cardiovascular disease, $N$ number, $R C T$ randomized controlled trial

models; 37,382 subjects); berries (6 models; 54,780 subjects); apples (5 models; 53,383 subjects); green tea (5 models; 25,420 subjects); cocoa-based products (4 models; 27,028 subjects); black tea (3 models; 42,699 subjects); monomer intake (3 models; 24,746 subjects); grape and raisins (3 models; 62,461 subjects); plums (3 models; 27,365 subjects); red wine (3 models; 5434 subjects); unspecified type of tea (2 models; 5399 subjects); 


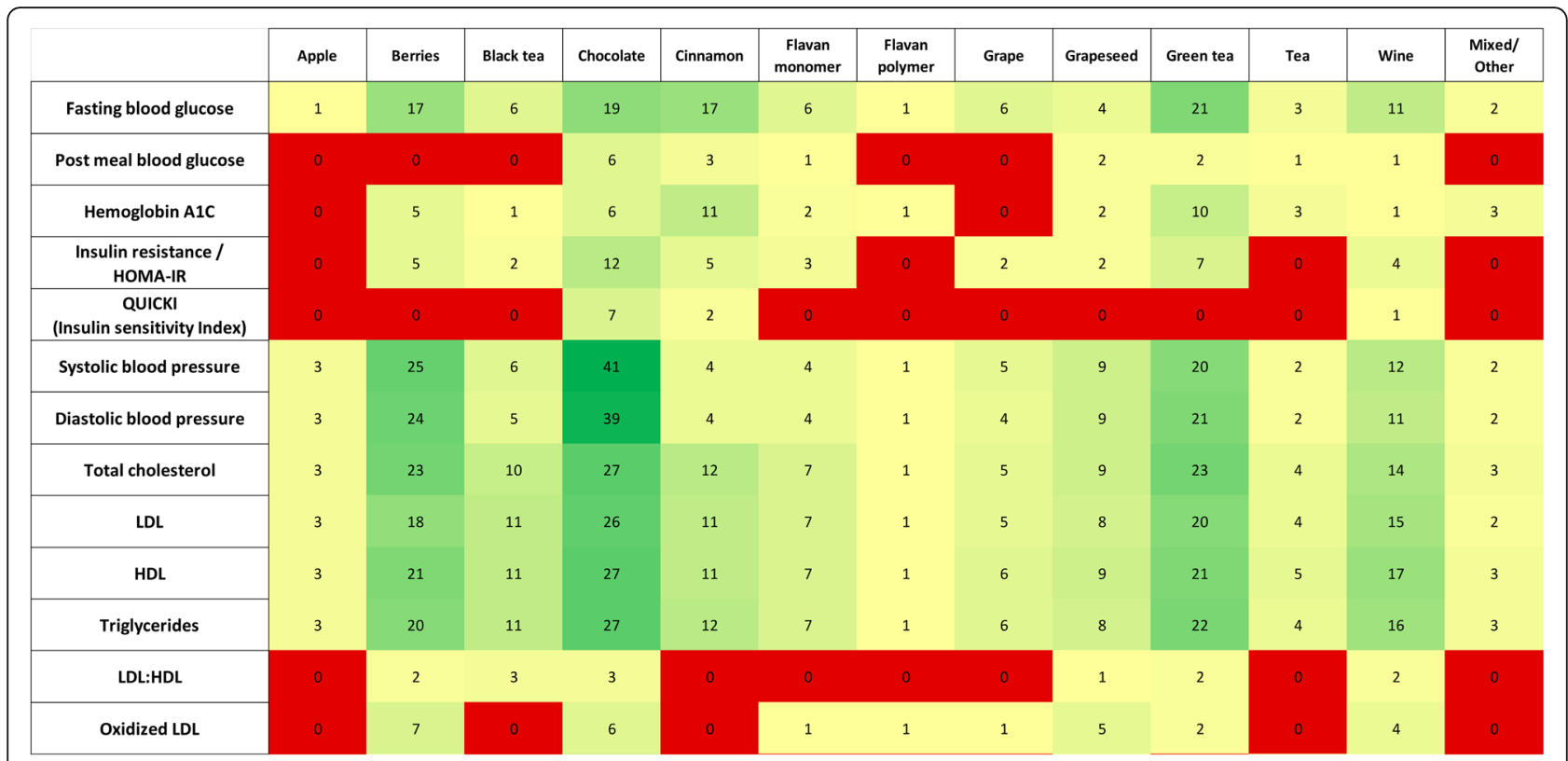

Fig. 4 Heat map showing the distribution of RCTs by their intervention arms and most commonly evaluated outcomes. RCTs = randomized controlled trials

and proanthocyanidin intake (1 model; 40,574 subjects). Across cohort studies, the average study duration ranged between 4.5 and 24 years. The associated cardiovascular outcomes examined included cardiovascular mortality, cerebrovascular stroke, and BP.

\section{Discussion}

This evidence mapping on flavan-3-ol intakes identified that cocoa-based products, tea, berries, and red wine each have several published RCTs examining relevant CVD and diabetes outcomes of interest. Additionally,

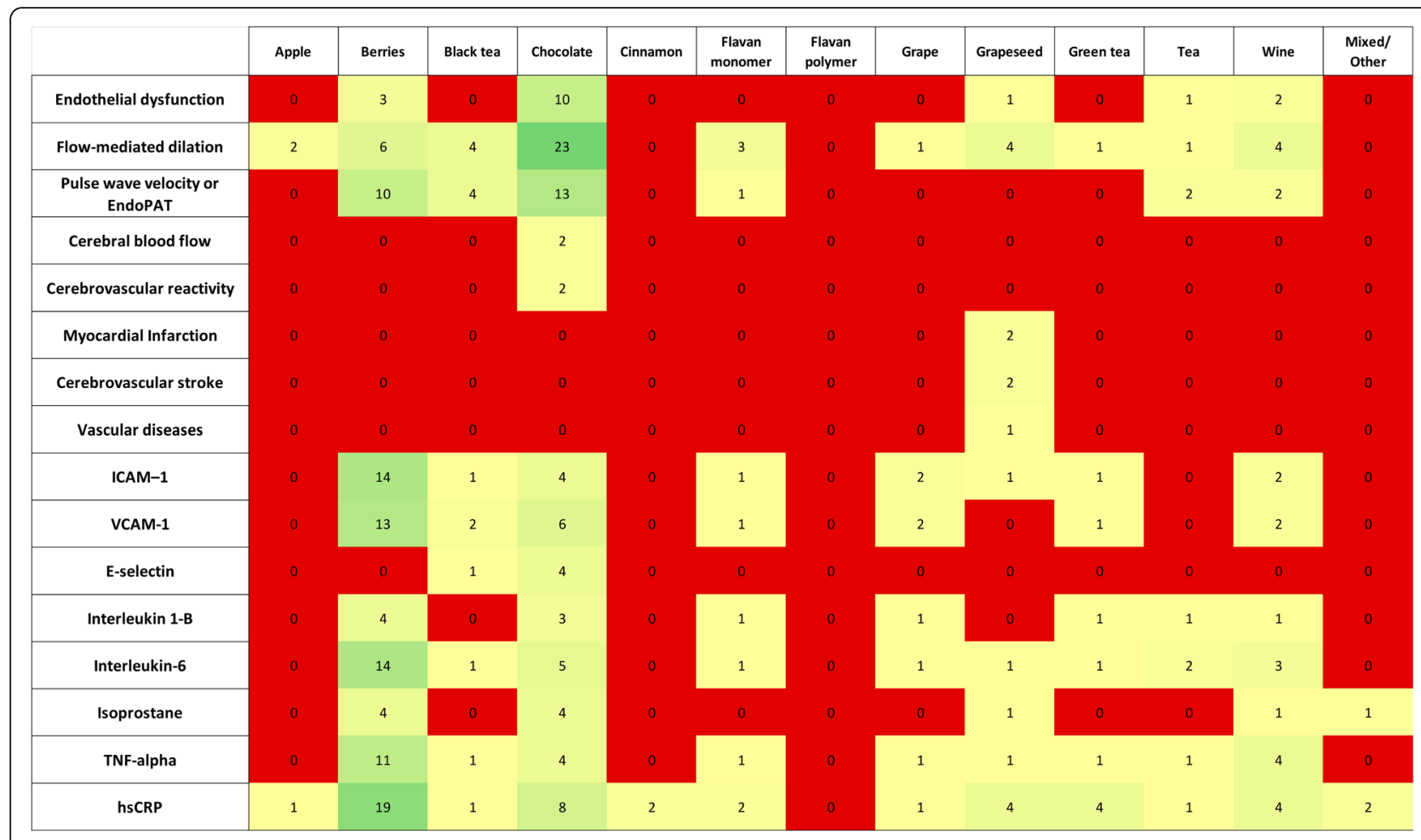

Fig. 5 Heat map showing the distribution of RCTs by their intervention arms and less frequently evaluated outcomes. RCTs = randomized controlled trials 
aside from an overall focus on total flavan-3-ol intake, berries, apples, green tea, and cocoa-based products were the predominant, habitual dietary sources.

\section{Description of existing literature}

A number of meta-analyses have been published on the existing population-based evidence for some of these foods individually, but none has been conducted evaluating a broad range of topics with a specific focus toward total flavan-3-ol intake $[4,6]$. A previous systematic review on chocolate, which included six cohort studies and one cross-sectional study, showed that higher intake was associated with a $37 \%(95 \%$ CI $10-46 \%)$ reduction in risk for CVD and 29\% (2-48\%) reduction in risk for stroke, compared with lower intake [4]. Largely based on the same studies, three other meta-analyses of population-based data reported relative risk (RR) reductions of between 13 and $25 \%$ for stroke [6] in the highest (median $62.9 \mathrm{~g} /$ week) versus the lowest $(0 \mathrm{~g} /$ week $)$ intake of chocolate. For tea, a recent meta-analysis of 22 prospective studies reported that a $3 \mathrm{cup} /$ day increase in consumption was associated with a reduction in risk of incident heart disease (RR, 0.73; 95\% CI 0.53-0.99), incident stroke (RR, 0.82; 95\% CI 0.73-0.92), and total mortality (RR, $0.76 ; 95 \%$ CI $0.63-0.91$ ), but had no significant effect on stroke mortality (RR, 0.93; 95\% CI 0.83-1.05) [8].

Three meta-analyses of RCTs $[7,19,20]$ that reviewed the impact of cocoa/chocolate consumption on cardiometabolic health from the available short duration ( $\leq 18$ weeks) studies reported similar effects, despite minor differences in inclusion criteria in two RCTs $[7,19]$. One meta-analysis reported a $-2.77 \mathrm{mmHg}$ (95\% CI $-4.72,-0.82 \mathrm{mmHg}, p=0.005)$ decrease in systolic BP and $-2.20 \mathrm{mmHg}(95 \% \mathrm{CI}-3.46,-0.93 \mathrm{mmHg}$, $p=0.006)$ reduction in diastolic BP following cocoa intake (17). The other systematic reviews, which had minor differences in inclusion criteria, reported similar effects $[7,19]$. The magnitude of associations are clinically relevant and of significant public health importance. For tea, one meta-analysis of 11 short-term RCTs (duration 1-26 weeks) observed reductions in systolic $\mathrm{BP}$ and diastolic BP [5], while another meta-analysis of 10 studies suggested that consumption of black tea significantly reduced LDL cholesterol concentrations but did not alter total or high density lipoprotein (HDL) cholesterol levels [21].

In relation to the specific associations between habitual intakes of flavan-3-ols and CVD, the most recent review of prospective cohort studies reported no association with coronary heart disease (CHD) mortality, CHD incidence, and stroke risk [22]. However, in one study, higher flavan-3-ol intake (monomers only) was associated with a $17 \%$ decrease in CVD mortality [23]. Two other studies, which included both monomers and other pro(antho)cyanidins, also reported a $66 \%$ decrease in atherosclerotic vascular disease mortality [24] and a 60\% decrease in CVD events and mortality [25]. In relation to procyanidin intake alone, a higher intake was associated with a 10-13\% decrease in CVD mortality in two studies $[23,26]$ and a $17 \%$ decrease in incident and fatal CHD in one study [27].

In relation to RCTs, to date, three systematic reviews have examined the potential effective dose of flavan-3-ols from the short-term trials on tea and cocoa. One observed a non-linear dose-response effect with a maximal effect observed at a "total polyphenol intake" of $500 \mathrm{mg}$ [7]. Another suggested that intakes of epicatechin $>50 \mathrm{mg}$ resulted in greater effects on systolic BP and diastolic BP, while FMD improved at all levels of epicatechin intake [1]. The most recent systematic review suggested that the acute and chronic FMD and BP responses for epicatechin, catechin, or procyanidin intakes were marginally greater for these individual constituents compared to total flavan-3-ol intake [28]. Furthermore, while there was no indication of a dose-response for BP, there were non-linear dose-response relationships observed for FMD, with a greater acute response observed at lower intake levels ( $<1 \mathrm{~g} /$ day procyanidins, $<200 \mathrm{mg} /$ day monomers) and a U-shaped response observed following chronic ingestion [28]. Several flavan-3-ol studies have also been conducted focusing on feeding chemically "pure" (-) - epicatechin to participants. Two recent studies administered $100 \mathrm{mg} /$ day of epicatechin to participants for 4 weeks. One reported no change in FMD or BP but did report improvements in insulin resistance [29], while the second also reported favorable effects on insulin resistance and improvements in both inflammatory and lipid biomarkers [30].

\section{Role and value of evidence mapping}

There is an increasing interest in these types of evidence mapping methods to create databases for identification of gaps or topics for future reviews [31, 32]. At the outset, we identified two experts from the flavan-3-ol research community and consulted with them throughout the review process. These experts assisted with the preliminary refinement of the $\mathrm{PI}(\mathrm{E}) \mathrm{COS}$ definitions, the analytic framework, and the review of evidence mapping results, and confirmed that the evidence mapping was thorough and comprehensive.

Prior to the review of abstracts and full-text articles, the review team received training on the screening process about how to identify relevant abstracts and articles using the pre-specified eligibility criteria. Key steps of this evidence mapping included (1) the determination of appropriate clinical and biochemical markers as outcomes of interest; (2) the use of appropriate duration of follow-up to assess each of the outcomes; (3) the review of abstracts and full-text articles for relevance from a broad range of 


\section{Author details}

${ }^{1}$ Tufts Center for Clinical Evidence Synthesis, Institute for Clinical Research and Health Policy Studies, Tufts Medical Center, Box 63, 800 Washington Street, Boston, MA 02111, USA. ${ }^{2}$ Tufts University Friedman School of Nutrition Science and Policy, 150 Harrison Ave, Boston, MA 02111, USA. ${ }^{3}$ Agricultural Research Service, U.S. Department of Agriculture, Beltsville Human Nutrition Research Center, Beltsville, MD, USA. ${ }^{4}$ Department of Nutrition and Preventive Medicine, Norwich Medical School, University of East Anglia, Norwich, UK.

Received: 5 December 2017 Accepted: 26 June 2018 Published online: 18 July 2018

\section{References}

1. Hooper L, Kroon PA, Rimm EB, Cohn JS, Harvey I, Le Cornu KA, et al. Flavonoids, flavonoid-rich foods, and cardiovascular risk: a meta-analysis of randomized controlled trials. Am J Clin Nutr. 2008;88:38-50.

2. Cassidy A, Minihane AM. The role of metabolism (and the microbiome) in defining the clinical efficacy of dietary flavonoids. Am J Clin Nutr. 2017;105:10-22.

3. Rodriguez-Mateos A, Vauzour D, Krueger CG, Shanmuganayagam D, Reed J, Calani L, et al. Bioavailability, bioactivity and impact on health of dietary flavonoids and related compounds: an update. Arch Toxicol. 2014;88:1803-53.

4. Buitrago-Lopez A, Sanderson J, Johnson L, Warnakula S, Wood A, Di AE, et al. Chocolate consumption and cardiometabolic disorders: systematic review and meta-analysis. BMJ. 2011;343:d4488.

5. Greyling A, Ras RT, Zock PL, Lorenz M, Hopman MT, Thijssen DH, et al. The effect of black tea on blood pressure: a systematic review with metaanalysis of randomized controlled trials. PLoS One. 2014;9:e103247.

6. Larsson SC, Virtamo J, Wolk A. Chocolate consumption and risk of stroke: a prospective cohort of men and meta-analysis. Neurology. 2012;79:1223-9.

7. Shrime MG, Bauer SR, MCDonald AC, Chowdhury NH, Coltart CE, Ding EL. Flavonoid-rich cocoa consumption affects multiple cardiovascular risk factors in a meta-analysis of short-term studies. J Nutr. 2011;141:1982-8.

8. Zhang C, Qin YY, Wei X, Yu FF, Zhou YH, He J. Tea consumption and risk of cardiovascular outcomes and total mortality: a systematic review and metaanalysis of prospective observational studies. Eur J Epidemiol. 2015;30:103-13.

9. Berends LM, van der Velpen V, Cassidy A. Flavan-3-ols, theobromine, and the effects of cocoa and chocolate on cardiometabolic risk factors. Curr Opin Lipidol. 2015;26:10-9.

10. Del RD, Rodriguez-Mateos A, Spencer JP, Tognolini M, Borges G, Crozier A. Dietary (poly)phenolics in human health: structures, bioavailability, and evidence of protective effects against chronic diseases. Antioxid Redox Signal. 2013;18:1818-92.

11. Ludwig IA, Clifford MN, Lean ME, Ashihara H, Crozier A. Coffee: biochemistry and potential impact on health. Food Funct. 2014;5:1695-717.

12. Sarma DN, Barrett ML, Chavez ML, Gardiner P, Ko R, Mahady GB, et al. Safety of green tea extracts : a systematic review by the US Pharmacopeia. Drug Saf. 2008:31:469-84.

13. Verhelst X, Burvenich P, Van SD, Gabriel C, Lootens M, Baert D. Acute hepatitis after treatment for hair loss with oral green tea extracts (Camellia Sinensis). Acta Gastroenterol Belg. 2009;72:262-4.

14. Bragge P, Clavisi O, Turner T, Tavender E, Collie A, Gruen RL. The global evidence mapping initiative: scoping research in broad topic areas. BMC Med Res Methodol. 2011;11:92.

15. Hetrick SE, Parker AG, Callahan P, Purcell R. Evidence mapping: illustrating an emerging methodology to improve evidence-based practice in youth mental health. J Eval Clin Pract. 2010;16:1025-30.

16. Bhagwat S, Haytowitz D. USDA's Database for the Proanthocyanidin Content of Selected Foods U.S. Department of Agriculture, Agricultural Service. Nutrient Data Laboratory Home Page. 2015. http://www.ars.usda. gov/nutrientdata/flav. Accessed 21 Nov 2017.

17. Bhagwat S, Haytowitz DB. USDA Database for the Flavonoid Content of Selected Foods, Release 3.2. U.S. Department of Agriculture, Agricultural Research Service. Nutrient Data Laboratory Home Page. 2015. http://www. ars.usda.gov/nutrientdata/flav. Accessed 21 Nov 2017.

18. Neveu V, Perez-Jiménez J, Vos F, Crespy V, du Chaffaut L, Mennen L, et al. Phenol-Explorer: an online comprehensive database on polyphenol contents in foods. 2017; https://doi.org/10.1093/database/bap024.

19. Hooper L, Kay C, Abdelhamid A, Kroon PA, Cohn JS, Rimm EB, et al. Effects of chocolate, cocoa, and flavan-3-ols on cardiovascular health: a systematic review and meta-analysis of randomized trials. Am J Clin Nutr. 2012;95:740-51.
20. Ried K, Sullivan TR, Fakler P, Frank OR, Stocks NP. Effect of cocoa on blood pressure. Cochrane Database Syst Rev. 2012;8:CD008893.

21. Zhao Y, Asimi S, Wu K, Zheng J, Li D. Black tea consumption and serum cholesterol concentration: systematic review and meta-analysis of randomized controlled trials. Clin Nutr. 2015;34:612-9.

22. Blumberg JB, Camesano TA, Cassidy A, Kris-Etherton P, Howell A, Manach C, et al. Cranberries and their bioactive constituents in human health. Adv Nutr. 2013:4:618-32.

23. McCullough ML, Peterson JJ, Patel R, Jacques PF, Shah R, Dwyer JT. Flavonoid intake and cardiovascular disease mortality in a prospective cohort of US adults. Am J Clin Nutr. 2012;95:454-64.

24. Ivey $\mathrm{KL}$, Lewis JR, Prince RL, Hodgson JM. Tea and non-tea flavonol intakes in relation to atherosclerotic vascular disease mortality in older women. Br J Nutr. 2013;110:1648-55.

25. Tresserra-Rimbau A, Medina-Remon A, Perez-Jimenez J, Martinez-Gonzalez MA, Covas MI, Corella D, et al. Dietary intake and major food sources of polyphenols in a Spanish population at high cardiovascular risk: the PREDIMED study. Nutr Metab Cardiovasc Dis. 2013;23:953-9.

26. Zamora-Ros R, Jimenez C, Cleries R, Agudo A, Sanchez MJ, SanchezCantalejo $\mathrm{E}$, et al. Dietary flavonoid and lignan intake and mortality in a Spanish cohort. Epidemiology. 2013;24:726-33.

27. Cassidy A, Mukamal KJ, Liu L, Franz M, Eliassen AH, Rimm EB. High anthocyanin intake is associated with a reduced risk of myocardial infarction in young and middle-aged women. Circulation. 2013;127:188-96.

28. Kay CD, Hooper L, Kroon PA, Rimm EB, Cassidy A. Relative impact of flavonoid composition, dose and structure on vascular function: a systematic review of randomised controlled trials of flavonoid-rich food products. Mol Nutr Food Res. 2012;56:1605-16.

29. Dower JI, Geleijnse JM, Gijsbers L, Zock PL, Kromhout D, Hollman PC. Effects of the pure flavonoids epicatechin and quercetin on vascular function and cardiometabolic health: a randomized, double-blind, placebo-controlled, crossover trial. Am J Clin Nutr. 2015;101:914-21.

30. Gutierrez-Salmean G, Meaney E, Lanaspa MA, Cicerchi C, Johnson RJ, Dugar $\mathrm{S}$, et al. A randomized, placebo-controlled, double-blind study on the effects of (-)-epicatechin on the triglyceride/HDLc ratio and cardiometabolic profile of subjects with hypertriglyceridemia: unique in vitro effects. Int J Cardiol. 2016:223:500-6.

31. Livingston KA, Chung M, Sawicki CM, Lyle BJ, Wang DD, Roberts SB, et al. Development of a publicly available, comprehensive database of fiber and health outcomes: rationale and methods. PLoS One. 2016;11:e0156961.

32. Wang DD, Shams-White M, Bright OJ, Parrott JS, Chung M. Creating a literature database of low-calorie sweeteners and health studies: evidence mapping. BMC Med Res Methodol. 2016;16:1.

\section{Ready to submit your research? Choose BMC and benefit from:}

- fast, convenient online submission

- thorough peer review by experienced researchers in your field

- rapid publication on acceptance

- support for research data, including large and complex data types

- gold Open Access which fosters wider collaboration and increased citations

- maximum visibility for your research: over $100 \mathrm{M}$ website views per year

At BMC, research is always in progress.

Learn more biomedcentral.com/submissions 